\title{
Immediate versus Delayed Contralateral Breast Symmetrisation in Breast Reconstruction with Latissimus dorsi Flap: A Comparative Study
}

\author{
Salvatore Giordano ${ }^{a}$ Sofia Harkkila ${ }^{a}$ Carlo M. Oranges ${ }^{b}$ Pietro G. di Summac \\ Ilkka Koskivuo ${ }^{a}$ \\ ${ }^{a}$ Department of Plastic and General Surgery, Turku University Hospital and the University of Turku, Turku, Finland; \\ ${ }^{b}$ Department of Plastic, Reconstructive, Aesthetic, and Hand Surgery, Basel University Hospital, University of Basel, \\ Basel, Switzerland; ' Department of Plastic, Reconstructive and Hand Surgery, University Hospital of Lausanne \\ (CHUV), Lausanne, Switzerland
}

\section{Keywords}

Latissimus dorsi - Breast reconstruction - Symmetrisation . Immediate symmetrisation - Delayed symmetrisation

\section{Abstract}

To achieve symmetry in unilateral free flap breast reconstruction often requires a contralateral procedure. There is no evidence in the literature to support the benefit of immediate contralateral breast symmetrisation concomitant to breast reconstruction. We hypothesized that performing a simultaneous contralateral balancing operation at the time as the initial reconstruction might provide immediate symmetry and minimize the frequency of secondary procedures. Thus, we performed a comparative study on this issue. A comparative retrospective study was conducted on 78 consecutive patients who underwent unilateral breast reconstruction surgery with latissimus dorsi (LD) flap and contralateral breast symmetrisation from January 2014 to June 2016 at Turku University Hospital. Exclusion criteria included other breast reconstruction techniques and no contralateral symmetrisation at follow-up. The patients were divided according to the timing of contralateral breast balancing operation into an immediate versus a delayed group. Postoperative complications, outcomes, and re-operations were compared. Baseline characteristics were well balanced between the groups except for comorbidity, which was significantly higher in the immediate group. Mastectomy weights (735.6 vs. $390.7 \mathrm{~g}, p=0.015$ ), contralateral breast reduction weights ( 268.3 vs. $105.8 \mathrm{~g}, p=0.014)$, and implant size (218.9
\end{abstract}

vs. $138.9 \mathrm{~g}, p=0.001$ ) were significantly larger in the immediate group. No significant differences in any kind of complications were detected. Similarly, the rates of re-operations were similar among the groups ( 24.0 vs. $43.3 \%, p=0.134$ ). Performing immediate symmetrisation at the time of breast reconstruction is safe and feasible in autologous LD breast reconstructions, where $76 \%$ did not require a second operation for symmetry. There were no differences in the rate of any re-operation and, therefore, performance of simultaneous contralateral reduction is a reasonable option.

(c) 2019 S. Karger AG, Basel

\section{Introduction}

The latissimus dorsi (LD) flap has been proven to be a very reliable and versatile method and is one of the best options for both immediate and delayed breast reconstructions with minimal donor site morbidity [1-4]. Beyond the consensus regarding this reconstructive procedure, its use has traditionally been limited by the desired size of the reconstructed and contralateral breast. To overcome any shortfall in final volume, the flap is very often routinely augmented at the time of its harvest by the positioning of breast implants or autologous fat [5]. To further improve the aesthetic outcomes, a contralateral balancing procedure may be offered including breast reduction, mastopexy, or augmentation to obtain symmetrical breast mounds [6]. Symmetrisation procedures of the contralateral healthy breast have become an essential

\section{KARGER}

๑) 2019 S. Karger AG, Basel 
Table 1. Demographics of the patients at the time of the study

\begin{tabular}{lccc}
\hline & $\begin{array}{l}\text { Immediate } \\
\text { group }(n=48)\end{array}$ & $\begin{array}{l}\text { Delayed } \\
\text { group }(n=30)\end{array}$ & $p$ value \\
\hline Mean age \pm SD, years & $55.0 \pm 7.5$ & $59.0 \pm 8.7$ & 0.130 \\
Mean BMI \pm SD, kg/m m $^{2}$ & $27.2 \pm 3.9$ & $26.9 \pm 6.4$ & 0.829 \\
Any comorbidity, $n(\%)$ & $35(73.9)$ & $22(73.3)$ & 0.975 \\
Diabetes, $n(\%)$ & $4(8.3)$ & $4(13.3)$ & 0.565 \\
HTA, $n(\%)$ & $13(27.1)$ & $12(40.0)$ & 0.341 \\
Lipid disease, $n(\%)$ & $6(12.5)$ & $8(26.7)$ & 0.190 \\
Depression, $n(\%)$ & $5(10.4)$ & $2(6.7)$ & 0.654 \\
Smokers, $n(\%)$ & $10(20.8)$ & $2(6.7)$ & 0.207 \\
BRCA, $n(\%)$ & $3(6.3)$ & $0(0.0)$ & 0.325 \\
Radiotherapy, $n(\%)$ & $22(78.6)$ & $18(60.0)$ & 0.394 \\
Chemotherapy, $n(\%)$ & $38(80.9)$ & $18(60.0)$ & 0.101 \\
\hline
\end{tabular}

part of post-mastectomy reconstructions, allowing the surgeon to reach the final goal of breast symmetry.

Even though contralateral procedures are performed to improve symmetry, an asymmetry between the 2 breasts might occurs over time because of the difference of the tissue between the natural breast parenchyma and the reconstructed breast. Despite its prevalence, there is limited literature that addresses the timing of the contralateral breast symmetrisation [7]. Possible advantages of immediate symmetrisation include a single operation and hospital admission with associated morbidity reductions and cost-savings for patients and healthcare providers, as well as reduced patients' distress due to breast asymmetry and the opportunity to sample contralateral breast tissue for occult malignancy $[5,7,8]$. On the other hand, immediate symmetrisation may result in more revisions and complications, even compromising further oncological treatments [7]. Nonetheless, ancillary breast procedures to achieve optimal aesthetic outcomes after primary reconstruction constitute a rising workload for any Plastic Surgery service [9].

Thus, the ideal timing of contralateral breast symmetrisation with unilateral breast reconstruction remains controversial. The aim of this study was to compare the revision and complication rates between immediate and delayed contralateral symmetry procedure in unilateral LD breast reconstruction. We hypothesized that performing an immediate symmetrisation at the time of breast reconstruction might reduce further procedures after autologous LD breast reconstructions.

\section{Materials and Methods}

This is a retrospective chart review of all consecutive patients who underwent unilateral breast reconstruction surgery using LD flap and any contralateral healthy breast reduction or mastopexy after mastectomy from January 2014 to June 2016 at Turku Uni- versity Hospital, Turku, Finland. All patients who underwent contralateral healthy breast symmetry procedures were included in this analysis. Thus, patients who underwent $\mathrm{LD}$ reconstruction but did not receive any contralateral breast symmetrisation procedures were excluded. The indications for LD breast reconstruction and contralateral healthy breast symmetrisation were at the discretion of the individual surgeon agreed with the patient. The patients were divided into 2 group according to the timing of contralateral healthy breast symmetrisation: immediate or delayed group.

The medical records were reviewed for demographics, comorbidities, treatment characteristics, including radio- and chemotherapy, timing of initial LD reconstruction, and timing and type of symmetry procedure performed. Postoperative complications included all complications that occurred in the delayed group during the initial LD flap procedure and, later, during the contralateral procedure, in order to assess and better quantify the differences between the 2 surgical strategies.

Nipple-areolar-complex reconstructions were not considered as surgical revisions because in our institution they are routinely performed under local anaesthesia at an outpatient setting. Similarly, minor scar and surgical revisions performed under local anaesthesia were excluded from the analysis. Therefore, all reported revisions on the contralateral healthy breast were performed in an operating room under general anaesthesia and included secondary mastopexy or reduction, implant exchange or removal, capsulotomy or capsulectomy, seroma, wound revisions, major dog ear excisions and scar revisions, and fat grafting.

Wound infection was defined as any surgical wound requiring antimicrobial treatment for superficial infection, emergency drainage, or hospital admission for deep infection. Wound dehiscence was defined as a wound breakdown with full-thickness skin separation extending $0.5 \mathrm{~cm}$ with or without infection resulting in delayed healing, $>2$ weeks, or demanding specialist dressing care. Seroma was defined as a collection of serous fluid or blood between tissues, identified through a clinical examination or ultrasound imaging and requiring percutaneous or operative drainage. Hematomas included haemorrhages requiring blood cell transfusion or emergency exploration in the operating room.

Our primary outcome measure was the rate of all-cause revision surgery between the groups. Secondary outcome measures included details of the operative technique (mastectomy weight, implant volume, reduction weight, and operative time), hospital stay, and return to work.

The results of parametric and non-parametric data were expressed as mean \pm SD, and SPSS statistical software (SPSS 23.0, Chicago, IL, USA) was used for all statistical analyses. Comparisons between the 2 groups were performed using the $\chi^{2}$ test or Fisher's exact test when appropriate. Continuous variables were compared using the analysis of variance test. CIs were set at $95 \%$. A two-sided $p$ value of $\leq 0.05$ was considered as statistically significant.

\section{Results}

Of 113 consecutive patients undergoing unilateral LD flap for breast reconstruction, 78 (69.0\%) patients underwent a contralateral symmetry procedure, immediately in 48 cases and delayed in the other 30 cases. Average patient age was 55.9 years (range $34-70$ years), and average BMI was $27.2\left(\right.$ range $\left.19-42 \mathrm{~kg} / \mathrm{m}^{2}\right)$. Table 1 outlines the 2 study groups, which did not present any significant differences 
Table 2. Comparison of perioperative parameters in the 2 groups of patients

\begin{tabular}{llcc}
\hline & $\begin{array}{l}\text { Immediate group } \\
(n=48)\end{array}$ & $\begin{array}{l}\text { Delayed group } \\
(n=30)\end{array}$ & $p$ value \\
\hline Operative time, min & $233.8 \pm 58.5$ & $212.6 \pm 63.7$ & 0.359 \\
Breast mastectomy resection weight, $g$ & $735.6 \pm 298.4$ & $390.7 \pm 136.5$ & 0.015 \\
Breast implant size (when used) & $218.9 \pm 67.0$ & $138.9 \pm 23.1$ & 0.001 \\
Breast reduction/mastopexy symmetrisation weight, g & $268.3 \pm 225.9$ & $105.8 \pm 140.9$ & 0.014 \\
Estimated blood loss, mL & $257.2 \pm 121.6$ & $259.1 \pm 207.1$ & 0.968 \\
Hospital stay, days & $4.2 \pm 1.1$ & $4.0 \pm 0.7$ & 0.572 \\
Follow-up, months & $34.7 \pm 18.2$ & $28.1 \pm 19.9$ & 0.258 \\
\hline
\end{tabular}

Table 3. Postoperative complications

\begin{tabular}{lccc}
\hline & $\begin{array}{c}\text { Immediate group } \\
(n=48), n(\%)\end{array}$ & $\begin{array}{c}\text { Delayed group } \\
(n=30), n(\%)\end{array}$ & $p$ value \\
\hline Complications & $20(41.6)$ & $18(60.0)$ & 0.163 \\
Superficial infection & $4(8.3)$ & $6(20.0)$ & 0.209 \\
Deep infection & $3(6.3)$ & $2(6.7)$ & 0.954 \\
LD donor site seroma (requiring drainage) & $12(25.0)$ & $8(26.6)$ & 0.878 \\
Haematoma (requiring intervention) & $1(2.1)$ & $0(0.0)$ & 1.000 \\
Wound dehiscence & $0(0.0)$ & $0(0.0)$ & 1.000 \\
Skin necrosis & $0(0.0)$ & $2(6.7)$ & 0.087 \\
\hline
\end{tabular}

LD, latissimus dorsi.

in demographics and pre-operative data; the 2 groups were well balanced. $75 \%$ of the patients required a breast implant together with LD flap.

Significant differences were detected in the implant size, breast mastectomy resection weight, and breast reduction/ mastopexy weight in the immediate symmetrisation group (Table 2). Other parameters, like estimated blood loss, length of hospital stay, and follow-up duration, were similar between the 2 groups. The mean time to the contralateral breast symmetrisation for the delayed group was $9.4 \pm$ 4.2 months from the initial breast reconstruction.

No significant differences were observed among overall complications nor among specific surgical site complications. Similarly, there were no significant differences among specific wound healing complications between the 2 groups (Table 3 ).

A total of 25 patients (32.0\%) required any kind of revision, $24 \%$ among the immediate group and $43.3 \%$ among the delayed group without any statistically significant differences, except for fat grafting revisions, which were higher in the delayed group. Most of the immediate group patients (76\%) did not require any revisions. Most of the re-operations involved the breast implant in the reconstructed side, with only one re-reduction contralateral breast procedure in the immediate group (Table 4).
Table 4. Re-operations (excluding the contralateral breast symmetrisation procedure in the delayed group)

\begin{tabular}{llll}
\hline & $\begin{array}{l}\text { Immediate group } \\
(n=48), n(\%)\end{array}$ & $\begin{array}{l}\text { Delayed group } \\
(n=30), n(\%)\end{array}$ & $p$ value \\
\hline Breast implant revision & $6(12.5)$ & $5(16.6)$ & 0.741 \\
Wound revision & $1(2.1)$ & $1(3.3)$ & 1.000 \\
Scar revision & $1(2.1)$ & $1(3.3)$ & 1.000 \\
Dog ear excision & $3(8.3)$ & $2(6.7)$ & 1.000 \\
Fat grafting & $0(0.0)$ & $4(13.3)$ & 0.019 \\
Re-reduction & $1(2.1)$ & $0(0.0)$ & 1.000 \\
\hline Total re-operations & $12(24.0)$ & $13(43.3)$ & 0.134 \\
\hline
\end{tabular}

Time until return to work for the active patients was not significantly different between the 2 groups $(26.9 \pm 18.2$ vs. $26.2 \pm 21.7$ days, $p=0.654$ ).

\section{Discussion/Conclusion}

Performing immediate symmetrisation at the time of breast reconstruction is a reasonable and safe option in autologous LD breast reconstructions, where $76 \%$ in our study 
did not require a second operation for contralateral breast symmetry. To achieve symmetry frequently requires a balancing procedure to the contralateral breast in unilateral breast reconstruction. In our study population, all contralateral procedures were breast reductions/mastopexy without any augmentation. It has been estimated that almost half of all patients (153/336) undergoing breast reconstruction as a part of breast cancer surgery had a symmetrisation procedure performed on the opposite side [9]. There is a relative lack of literature examining the treatment of the contralateral breast, particularly in a comparative way. Some authors have reported on contralateral symmetry procedures in the immediate setting, or delayed, in conjunction with revisions to the index reconstruction or donor site and have demonstrated promising results [6-14].

The main limiting factor in autologous breast reconstruction is the volume of donor site tissue, and when as much healthy tissue as possible is transferred, the contralateral breast may need to be reduced or lifted to achieve size match. In our study, we found that the size of the breast implant was significantly smaller in the delayed group indicating that the operating surgeon did not initially schedule to perform any contralateral procedure because they probably did not find a clinically significant breast size difference at the time of the initial reconstruction. Conversely, the initial mastectomy weight and the healthy breast reduction/mastopexy resections were significantly higher in the immediate symmetrisation group, showing that patients with large breasts are more likely to need a contralateral procedure (Table 2).

Contrary to Chang et al.'s studies [7, 11], immediate symmetry procedures did not result in twice as many revisions and complications as delayed procedures. However, in their study [11], 65\% of the patients undergoing an immediate symmetry procedure did not require any further procedures; and this rate is similar to our finding (76\%). In our study, we found that the rates of fat grafting revisions were significantly higher in the delayed symmetrisation group.

Performing a simultaneous reduction using the reconstructed breast as a model for the contralateral breast is a very feasible way to achieve promising aesthetic results and patients' satisfaction reducing the number of further procedures. Intuitively, patients undergoing an immediate contralateral balancing procedure have fewer operations, with obvious implications on the health-care costs, use of resources, need for another general anaesthesia, and longer recovery.

Contrary to the common belief that the contralateral breast should not be symmetrised at the time of reconstruction as the flap should be given time to "settle" before the surgeon attempts to match the native breast, we found a significantly higher rate $(43.3 \%)$ of revision surgery in patients undergoing delayed contralateral symmetrisa- tion. It is possible that women who have their mastectomy, reconstruction, and symmetrisation as one single operation may be more satisfied with the surgical outcome [8] and, thus, are less likely to consult the surgeon again for revision surgery. Supporting this hypothesis, Yip et al. [15] showed that in the context of reconstruction and contralateral symmetrisation, breast volume symmetry was not related to satisfaction, but that satisfaction was most influenced by the pre-operative care as part of the reconstructive treatment process. Nevertheless, several authors have demonstrated better or comparable satisfaction and aesthetic outcomes with immediate symmetrisation $[6,8$, $10,13]$. Due to the lack of data, we did not assess patients' satisfaction through patient-reported outcome measures.

On the other hand, a delayed approach involves more surgeries, which may damage patients' psychosocial wellbeing; affect work and life commitments; and increase clinic demand, operative time, hospital bed occupancy, length of surgical waiting lists, and follow-up appointments.

We did not find significant differences in operative time in the immediate versus delayed group, although the balancing procedure increased the duration of surgery by a mean of $20 \mathrm{~min}$, and hospital stay was also similar between the study groups.

The strengths of this study include a consistent surgical technique, long-term follow-up, and very comparable groups in terms of perioperative parameters and comorbidities. Several limitations of this study should be acknowledged. This is a retrospective study and the major drawback is due to the retrospective nature and small number of participants. Moreover, the lack of randomization and decision of the individual surgeon to perform immediate or delayed contralateral symmetrisation introduce the possibility of selection bias. Nonetheless, the decision to undergo a revision is not a direct objective measure of asymmetry. Furthermore, several non-clinical factors can possibly contribute to the timing and number of revisions, including not only surgeon's preferences, but also patients' preferences, financial and working issues, and timing of adjuvant therapies.

In conclusion, restoring symmetry is an important component of breast reconstruction. A contralateral symmetry procedure is often necessary in case of unilateral breast reconstruction. Performing immediate symmetrisation at the time of breast reconstruction may lead to a similar revision rate as delayed procedures, without a higher overall complication rate.

\section{Statement of Ethics}

This research complies with the guidelines for human studies and was conducted ethically in accordance with the World Medical Association Declaration of Helsinki. All subjects (or their par- 
ents or guardians) have given their written informed consent, and the study protocol was approved by the institute's Committee on Human Research. Ethical approval was not required because of the retrospective nature of this study.

\section{Disclosure Statement}

The authors have no conflicts of interest to declare.

\section{Funding Sources}

None.

\section{References}

1 Schneider WJ, Hill HL Jr, Brown RG. Latissimus dorsi myocutaneous flap for breast reconstruction. Br J Plast Surg. 1977 Oct;30(4): $277-81$.

2 Kääriäinen M, Giordano S, Kauhanen S, Lääperi AL, Mattila P, Helminen M, et al. The significance of latissimus dorsi flap innervation in delayed breast reconstruction: a prospective randomized study-magnetic resonance imaging and histologic findings. Plast Reconstr Surg. 2011 Dec;128(6):637e-45e.

3 Giordano S, Kääriäinen K, Alavaikko J, Kaistila T, Kuokkanen H. Latissimus dorsi free flap harvesting may affect the shoulder joint in long run. Scand J Surg. 2011;100(3):202-7.

4 Mushin OP, Myers PL, Langstein HN. Indications and Controversies for Complete and Implant-Enhanced Latissimus Dorsi Breast Reconstructions. Clin Plast Surg. 2018 Jan; 45(1):75-81.

5 Leuzzi S, Stivala A, Shaff JB, Maroccia A, Rausky J, Revol M, et al. Latissimus dorsi breast reconstruction with or without implants: A comparison between outcome and patient satisfaction. J Plast Reconstr Aesthet Surg. 2019 Mar;72(3):381-93.

\section{Author Contributions}

All authors: conception and design of the work. S.G. and S.H.: drafting the manuscript. All authors: critical review of the manuscript for important intellectual content. C.M.O., P.G.S., and I.K.: manuscript supervision. All authors: approval of the version to be published. We certify that all authors of this manuscript have participated in conceptualizing the research or content of the manuscript, in writing or critically editing the manuscript, and/or in analysis of data presented in the manuscript. Consent to submit has been received from all co-authors.
6 Inbal A, Gur E, Otremski E, Zaretski A, Amir A, Weiss J, et al. Simultaneous contralateral breast adjustment in unilateral deep inferior epigastric perforator breast reconstruction. J Reconstr Microsurg. 2012 Jun;28(5):285-92.

7 Chang EI, Selber JC, Chang EI, Nosrati N, Zhang H, Robb GL, et al. Choosing the optimal timing for contralateral symmetry procedures after unilateral free flap breast reconstruction. Ann Plast Surg. 2015 Jan;74(1):126.

8 Huang JJ, Wu CW, Leon Lam W, et al. Simultaneous contralateral breast reduction/mastopexy with unilateral breast reconstruction using free abdominal flaps. Ann Plast Surg. 2011 Oct; 67(4):336-42.

9 Mathew J, Courtney CA. Symmetrisation procedures in breast reconstruction patients constitute a rising workload in our DGH. Surgeon. 2014 Dec;12(6):307-9.

10 Ulusal BG, Cheng MH, Wei FC. Simultaneous endoscope-assisted contralateral breast augmentation with implants in patients undergoing postmastectomy breast reconstruction with abdominal flaps. Plast Reconstr Surg. 2006 Nov;118(6):1293-302.
11 Chang EI, Lamaris G, Chang DW. Simultaneous contralateral reduction mammoplasty or mastopexy during unilateral free flap breast reconstruction. Ann Plast Surg. 2013 Aug; 71(2):144-8.

12 Pauchot J, Feuvrier D, Panouilleres M, Pluvy I, Tropet Y. Benefit of simultaneous contralateral breast symmetry procedure with unilateral breast reconstruction using DIEP flaps. About 33 cases. Ann Chir Plast Esthet. 2015 Dec;60(6):472-7.

13 Laporta R, Longo B, Sorotos M, Pagnoni M, Santanelli Di Pompeo F. One-stage DIEP flap breast reconstruction: algorithm for immediate contralateral symmetrization. Microsurgery. 2016 Jan;36(1):7-19.

14 Wade RG, Marongiu F, Sassoon EM, Haywood RM, Ali RS, Figus A. Contralateral breast symmetrisation in unilateral DIEP flap breast reconstruction. J Plast Reconstr Aesthet Surg. 2016 Oct; 69(10):1363-73.

15 Yip JM, Watson DI, Tiggemann M, Hsia S, Smallman AE, Dean NR. Determinants of breast reconstruction outcome: how important is volume symmetry? J Plast Reconstr Aesthet Surg. 2015 May;68(5):679-85. 